\title{
Development and testing of braking and acceleration features for vehicle advanced driver assistance system
}

\author{
Johann Carlo Marasigan', Gian Paolo Mayuga ${ }^{1}$, Elmer Magsino² \\ 'Electronics, Computer and Communications Engineering Department, School of Science and Engineering, \\ Ateneo de Manila University, Quezon City, Philippines \\ ${ }^{2}$ Electronics and Communications Engineering Department, Gokongwei College of Engineering, De La Salle University, \\ Manila, Philippines
}

\section{Article Info \\ Article history: \\ Received May 14, 2021 \\ Revised Sep 15, 2021 \\ Accepted Oct 10, 2021}

\section{Keywords:}

Advanced driver assistance

Brake and acceleration features

Fuzzy logic

Intelligent transportation

systems

Phantom traffic jam

System

\begin{abstract}
Traffic congestion is a constant problem for cities worldwide. The human driving inefficiency and poor urban planning and development contribute to traffic buildup and travel discomfort. An example of human inefficiency is the phantom traffic jam, which is caused by unnecessary braking, causing traffic to slow down, and eventually coming to a stop. In this study, a brake and acceleration feature (BAF) for the advanced driver assistance system (ADAS) is proposed to mitigate the effects of the phantom traffic phenomenon. In its initial stage, the BAF provides a heads-up display that gives information on how much braking and acceleration input is needed to maintain smooth driving conditions, i.e., without sudden acceleration or deceleration, while observing a safe distance from the vehicle in front. BAF employs a fuzzy logic controller that takes distance information from a light detection and ranging (LIDAR) sensor and the vehicle's instantaneous speed from the engine control unit (ECU). It then calculates the corresponding percentage value of needed acceleration and braking in order to maintain travel objectives of smooth and safe-distance travel. Empirical results show that the system suggests acceleration and braking values slightly higher than the driver's actual inputs and can achieve $90 \%$ accuracy overall.
\end{abstract}

This is an open access article under the CC BY-SA license.

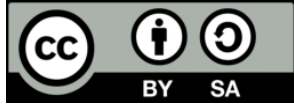

\section{Corresponding Author:}

Elmer Magsino

Electronics and Communications Engineering Department, Gokongwei College of Engineering,

De La Salle University

Manila, Philippines

Email: elmer.magsino@dlsu.edu.ph

\section{INTRODUCTION}

Traffic congestions are caused by the combination of poor urban planning and development, and inefficiency of human driving skills. An example of poor urban planning and development is the presence of bottlenecks [1], wherein multiple lanes compress into fewer lanes, forcing traffic to merge into its adjacent lanes thus, effectively slowing down traffic flow. On the other hand, human driving skill inefficiency causes phantom traffic jams. Phantom traffic jams [2] are mainly caused by the non-uniform flow of vehicles and their unequal spacing. When a vehicle slows down, a domino effect of decelerating vehicles behind them occurs, forcing traffic to effectively come to a halt. Unequal spacing is usually caused by tailgating, thereby, allowing the driver to misjudge its decisions based on the minimum required safe distance between two vehicles. Another factor of phantom traffic jams is the frequent lane changing because leading vehicles do not give way to faster trailing cars [3]. 
To mitigate the phantom traffic jam phenomenon, car manufacturers developed autonomous vehicles as well as technologies like adaptive cruise control (ACC) [4], lane keep assist [5] and automatic braking [6] which are designed to intervene with the driver's inputs when necessary. Autonomous vehicle technology is classified into six levels of autonomy [7]. Level 0 offers absolutely no form of automation, i.e., the control of the vehicle solely depends on the driver. Level 1, known as driver assistance, includes technologies that assist the driver in controlling the vehicle, such as conventional cruise control [8], adaptive cruise control, lane keep assist [9], [10] and automatic braking [11], [12]. Level 2 [13] offers partial automation where two or more automated systems function together to relieve the driver of control. However, the driver must still maintain their full attention on the road. Level 3 [14] is labeled as conditional automation where steering, acceleration and deceleration are controlled with regards to the driving environment. Level 4 [15] is known as high automation allows the vehicle full control of itself under certain conditions where human intervention is no longer needed, but human drivers can still take control of the vehicle. Level 5 [16] is the full automation of a vehicle and it does not permit human drivers to intervene. [17], [18].

In [19], findings showed that human behaviors tend to display opposite of what is necessary to eliminate traffic congestions. Simulations were done in an intelligent-driver model (IDM) microscopic traffic flow model that simulate simple human driving behavior as well as adaptive cruise control capabilities. To address phantom traffic jams, vehicles operating from Levels 1-5 have ACC that can automatically adjust the vehicle's speed and desired safe distance depending on the presence of a leading vehicle. Various linear and non-linear models and controller designs have been presented and compared to achieve the target ACC objectives [20]. As a result, the vehicular spacing is increased or decreased when its speed is reduced or escalated, respectively. Other ACC features included speed sign detection to monitor the allowable road speed limit and avoid speeding violations [21]. In [22], proper navigation tools were improved by considering the presence of inaccurate data and unavailability navigational tools.

ACC gives the driver the ability to set both a desired following distance and cruising speed. During velocity control, the vehicle does not detect any obstacle in front and continues to cruise at a constant and preset speed, however, when an obstacle is detected, ACC reverts to the distance control. The smooth switching between the speed and distance control can be accomplished by a hierarchical control [23]. When implementing ACC, potentially induced degradation (PID) [24], [25] and fuzzy logic [26]-[28] are the widely used control approaches. In [29], velocity control was achieved by implementing a PID controller, while a fuzzy controller was used for distance control. The test car was a 1993 Mitsubishi Galant with an automatic transmission where its engine and drive train were modified.

However, ACC systems were found to worsen the occurrence of phantom traffic jams as empirically proven by researchers from Vanderbilt University. A fleet of eight identical vehicles all equipped with ACC were used. They were driven for more than 1,200 miles to analyze the effects of this feature in high traffic density. In the experiment setup, the lead vehicle reduced its speed by $6 \mathrm{mph}$. As an effect, the trailing vehicles slowed down much more than the lead vehicle did. In fact, the last car's speed even dropped below the minimum speed required for the ACC system to function [30].

This study focuses on mitigating phantom traffic jams by developing a brake and acceleration feature (BAF) system that displays the required braking and acceleration inputs to maintain a safe intervehicle distance, specifically for older vehicle models, which make up majority of the vehicles in the Philippines. BAF is a real-time system that employs a fuzzy logic controller that accesses the vehicle's engine control unit (ECU) and light detection and ranging (LIDAR) sensor to calculate the appropriate driving conditions. The advantages of employing the developed BAF system are: i) it is easily connected to the vehicle's ECU and does not require any additional hardware, ii) conventional smart phones can be used as displays, therefore, automatically providing wireless access, portability, and transferability, and iii) BAF is adaptive to the driver's behavior due to the utilization of the fuzzy logic concept in understanding the needs and strategies of the driver. The outputs are displayed on a heads-up display (HUD) easily visible to the driver. BAF also focuses only on the vehicle directly in front of the host. Empirical experiments and testing have been implemented on a 2008 Toyota Vios 1.3J. No other modifications have been done to the vehicle's engine or drivetrain characteristics in order to quickly allow system integration with other car models. With the addition of the proposed BAF system, the vehicle falls under the Level 1 autonomous vehicle classification.

This work utilizes the fuzzy logic concept in order to address the dissimilarities on driver behavior and adaptability. Deligianni et al. [31] studied the naturalistic driving attitude in order to improve the comfortability of passengers riding autonomous vehicles. Based on these driving characteristics, linear or non-linear membership functions will be assigned to the BAF system accordingly with the main goal of mimicking the driving behavior. Given the natural vagueness of the driver's behaviors, fuzzy logic can easily address these indistinct and hard to separate driving features, e.g., acceleration and braking, hard or soft 
turning. This paper is organized as follows: section 2 shows the development of the HUD system and its installation in the test vehicle. The fuzzy logic membership functions and control surfaces used are also explained in this section. Section 3 shows the results of actual road testing the system with graphs to visualize and compare the outputs of the BAF and the actual inputs of the driver. The paper concludes in section 4, stating the findings, as well as recommendations on how the system may be improved in terms of design and functionality.

\section{DEVELOPMENT OF THE BRAKE AND ACCELERATION FEATURE SYSTEM}

In this section, we present the off-the-shelf components used in developing the hardware unit of the BAF system. We also discuss how these components are installed in a vehicle and how the BAF system is implemented.

\subsection{BAF system hardware}

Figure 1(a) shows the block diagram and proposed changes to a test vehicle while Figure 1(b) enumerates the actual components employed in developing the hardware unit of the BAF system. The LIDAR sensor used is a Garmin LIDAR-Lite V3 with a range of 5-40 meters. It operates on the I2C protocol that is easily interfaceable with a microcontroller and the Raspberry Pi 4. The Arduino's serial port reads the distance sensed by the LIDAR sensor. We note that for the meantime, the LIDAR is only utilized as a proximity sensor. To measure the vehicle's speed, BAF used a generic onboard diagnostics (OBD)-II adapter equipped with the ELM327 chip that enables interfacing with smartphones or computers through Wi-Fi, Bluetooth or USB. Both the Arduino and OBD adapter are then connected to the Raspberry Pi through USB. While the OBD-II can provide data on how much throttle input is being applied through the throttle position sensor, it cannot however, display how much braking force is being applied. To resolve this, a G-force meter, using the application physics toolbox suite on android is used to measure and log how much force the vehicle is experiencing under different braking intensities.

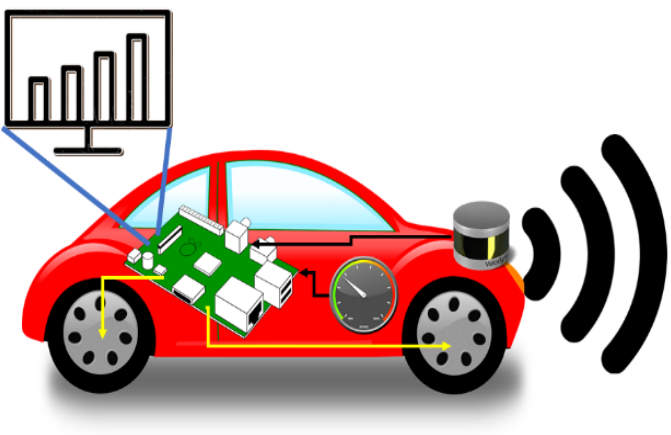

(a)

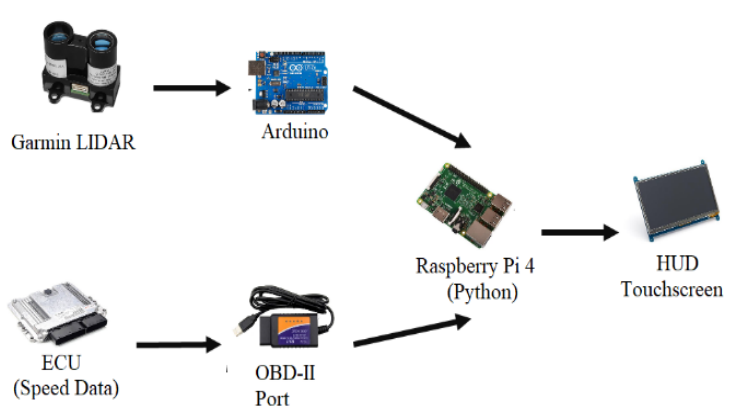

(b)

Figure 1. Brake and acceleration feature hardware (a) block diagram and (b) off-the-shelf sensors, electronic equipment, and its connection

\subsection{Installation of the $\mathbf{B A F}$ system in a vehicle}

Figure 2 shows the implementation of the proposed BAF system in a vehicle. Figure 2(a) illustrates the 2008 Toyota Vios 1.3J vehicle where the BAF system is installed. The LIDAR sensor and Arduino are placed outside the windshield. The sensor was originally intended to be mounted at the center of the dashboard. However, doing this resulted in the sensor continuously outputting one $\mathrm{cm}$. Because of this, the sensor is mounted outside the vehicle as shown in Figure 2(b). It is possible that the tint on the wind shield caused the erroneous readings as the signal emitted by the sensor could have been reflected by the dark shade instead of penetrating through it. The onboard diagnostics (OBD) adapter, Raspberry Pi, Heads-Up Display, and G-force measuring smartphone are installed inside the vehicle as seen from Figure 2(c). We note that there are no major changes introduced to the vehicle upon the installation of the BAF hardware system and is guaranteed to be applicable to any vehicle model as long as the vehicle's ECU is easily accessible.

\subsection{BAF system algorithm}

Having the Raspberry Pi as the main controller, the BAF algorithm is developed in Python to access the vehicle's ECU, to fetch the LIDAR sensor data through the Arduino serial output, to calculate the 
acceleration and braking values using fuzzy logic, and to log the necessary data and save in CSV format. The BAF System employs fuzzy logic because it can be adjusted according to the driver's behavior and/or vehicle's characteristics. As an initial assumption and for simplicity, linear membership functions are first utilized in the modeling of the human driving behavior. Such linear function can represent the linear acceleration and deceleration attitude of a driver who thinks of the passenger's comfort and convenience. A linear attitude can also be attributed to the slow turning on an intersection or when the vehicle is overtaking a slow car in front of it on a highway.

We classify the speed and distance inputs into five levels each and then determine whether to output an acceleration (A) or braking (B) value. In practical application, the number of classifications can be easily adjusted depending on the needs of the driver. Increasing the number of levels mean that the driver needs much assistance when compared to fewer levels of classifications. While there are two outputs, only one of the two will have a value greater than zero while the other exactly zero, as it would not make sense for a driver to step on the throttle and brake pedals at the same time. This assumes the normal urban driving conditions such as not too much sloped or elevated roads.

The speed range (S) is defined from 0 to $100 \mathrm{kph}$ (assuming also highway roads) and is classified into the five levels: too slow (TS), slow (S), normal (N), fast (F), and too fast (TF). Figure 3(a) shows the membership functions used to divide the speed range into its corresponding speed input interval. On the other hand, the distance range (D) from 0 to $3000 \mathrm{~cm}$ is categorized into another five levels: too close (TC), close (C), adequate (Adeq), far (F), and very far (VF). The input distance value is set to centimeters to increase precision. For a more sensitive response, the distance measure can still be reduced to millimeters. Figure 3(b) depicts the distance input separated into its equivalent definitions. The acceleration and braking responses $(\mathrm{ABR})$ of the BAF system are grouped into four levels, namely: light $(\mathrm{L})$, moderate $(\mathrm{M})$, heavy $(\mathrm{H})$, and full (F). These projected outputs from each scenario are based on the light to moderate traffic volume scenarios are shown in Figure 4.

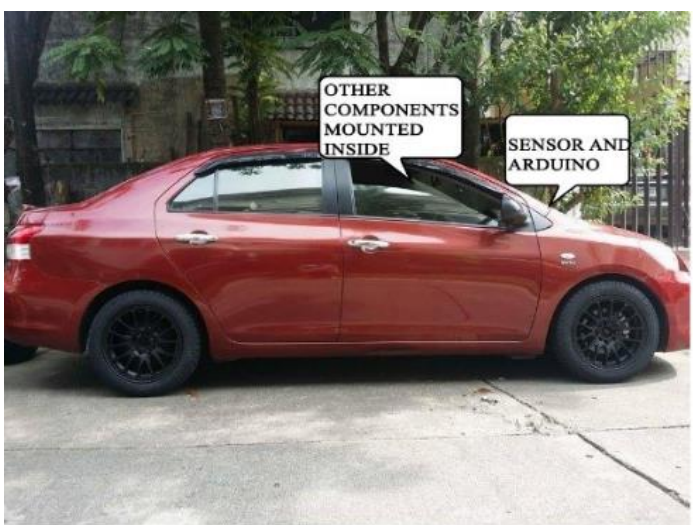

(a)

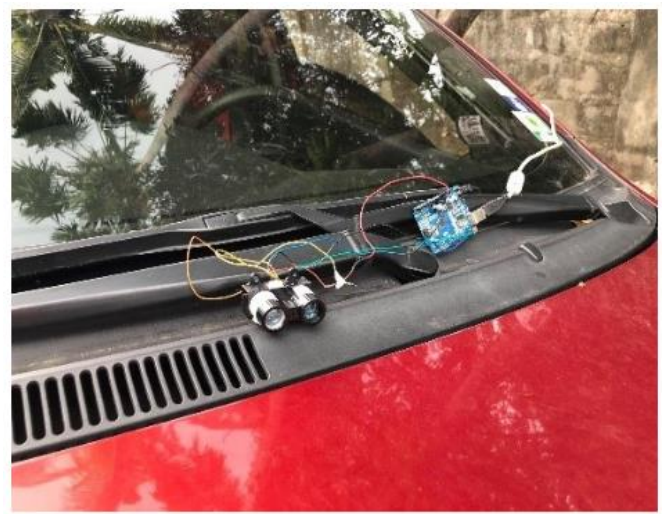

(b)

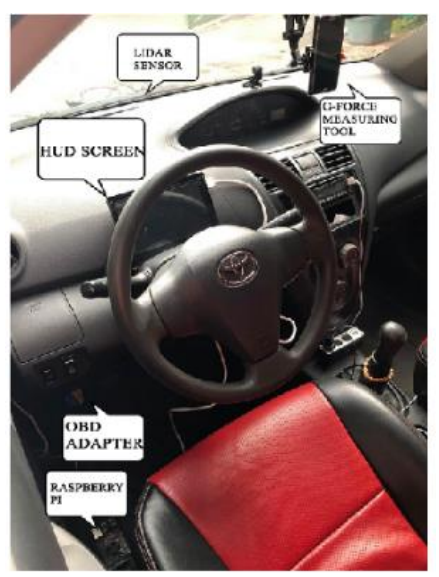

(c)

Figure 2. BAF implementation (a) The 2008 Toyota Vios 1.3J used in the experiments, (b) LIDAR sensor and Arduino, and (c) Interior Sensor Installation 


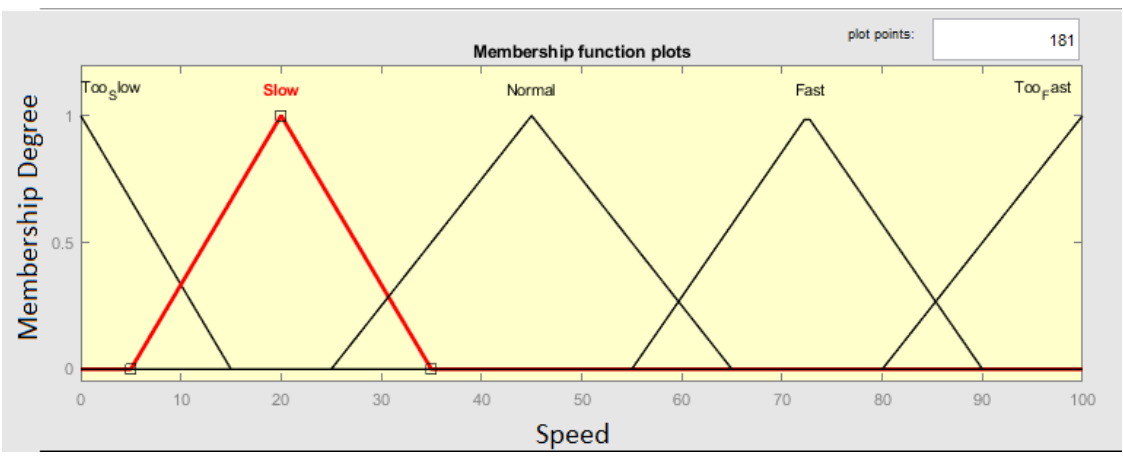

(a)

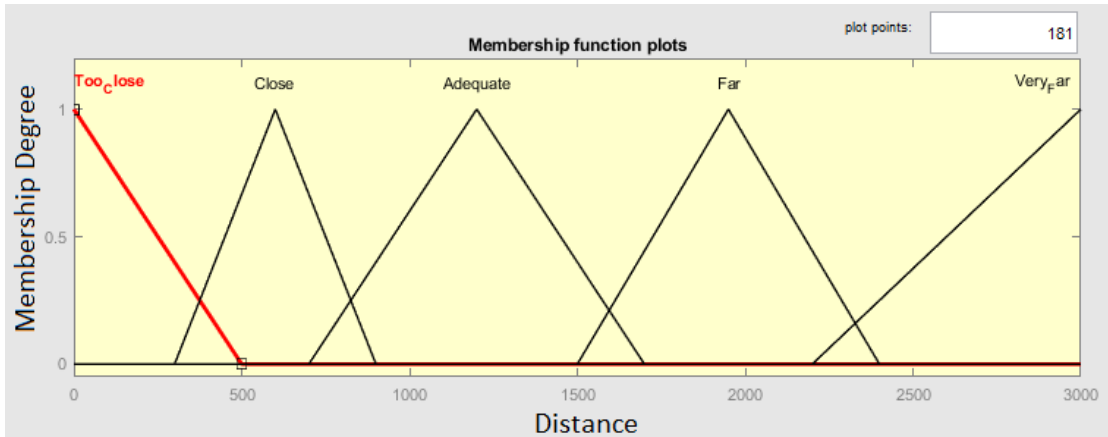

(b)

Figure 3. Triangular membership functions with varying base width model the fuzzy definition of the (a) vehicle's speed, and (b) vehicle's distance from the leading vehicle

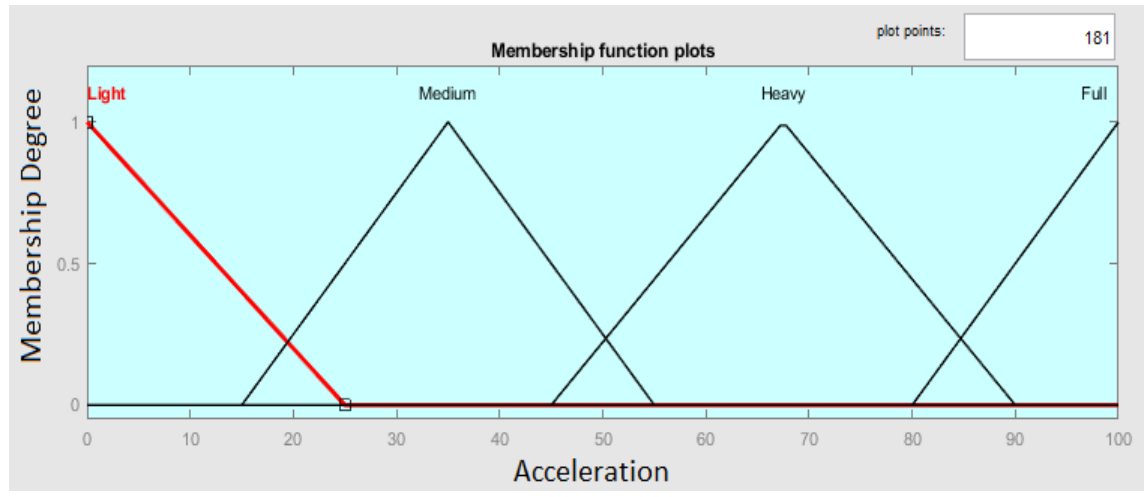

Figure 4. Triangular membership functions with varying base width model the fuzzy definition of the vehicle's acceleration and braking responses

The implementation of a fuzzy rule follows the general definition of: $A B R=f u z z y(S, D)$. Given the number of inputs, there are 25 possible ABR to be defined in the fuzzy rules. Table 1 shows the fuzzy rule base according to the definitions. For examples in Table 1, we have: i) if speed is NORMAL AND the distance is ADEQUATE, then acceleration is LIGHT (grey-colored cell) and ii) if speed is FAST and distance is $C L O S E$, then braking is MEDIUM (black-colored cell).

Table 1. Fuzzy rule base

\begin{tabular}{cccccc}
\hline SpeedLDistance & TC & C & A & F & VF \\
\hline TS & HB & MB & MA & HA & FA \\
S & HB & MB & LA & MA & HA \\
N & HB & MB & LA & LA & HA \\
F & FB & MB & LB & LA & LA \\
TF & FB & HB & MB & MB & MB \\
\hline
\end{tabular}

Development and testing of braking and acceleration features for vehicle ... (Johann Carlo Marasigan) 
In the first example, if the instantaneous speed is within the "normal" range and the inter-vehicle distance is within the "adequate" range, the driver will only have to apply light acceleration to maintain their current speed and inter-vehicle distance. In the second statement, given the speed value higher than the normal instantaneous speed, and closer than normal inter-vehicle distance, the driver would have to input a "medium" amount of braking to increase the inter-vehicle distance. Heavy braking may be applied but it may be unnecessary. To immediately visualize the input-output fuzzy control relationships, the surface views of the fuzzy rule base in Table 1 are shown in Figures 5(a) and 5(b).

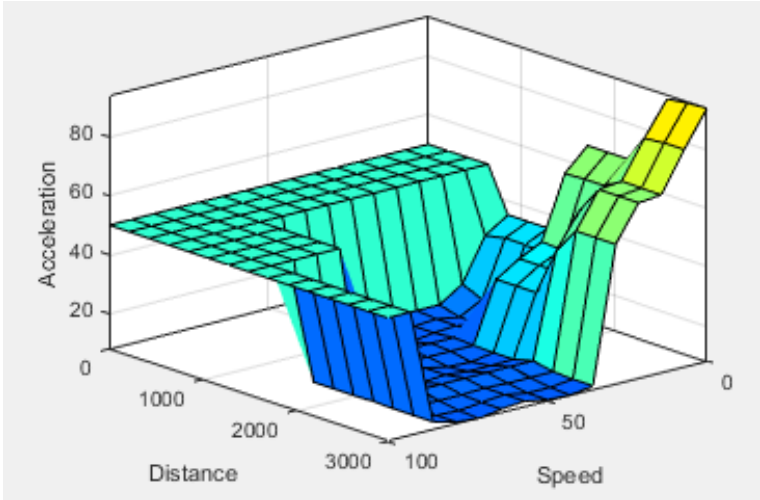

(a)

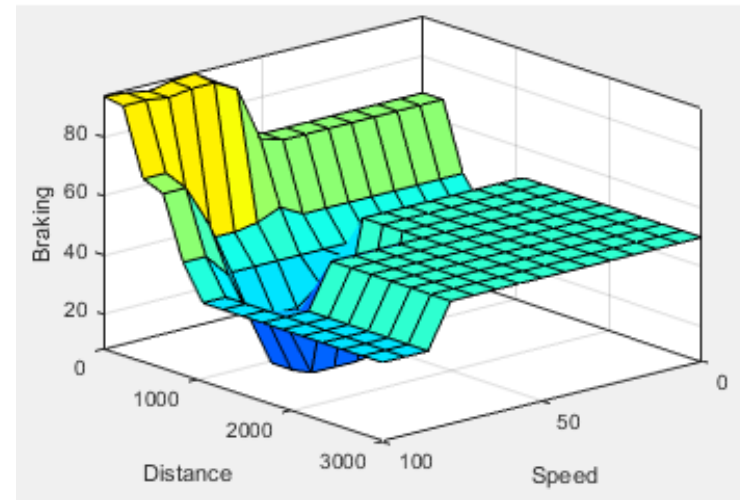

(b)

Figure 5. Speed/distance, (a) acceleration, and (b) braking fuzzy relationship

Just like in [32], the fuzzy logic controller's implication method employed the min operator, while the outputs are aggregated by using the max operator. The defuzzification is achieved by calculating the centroid of the aggregated output. We note that both acceleration and braking responses will always have numerical values. Given this, the BAF decides on utilizing the response with a bigger fuzzy output value. Once a satisfactory fuzzy control response is achieved, these rules are then setup in the Python IDE by using the scikit-fuzzy library.

\section{RESULTS AND DISCUSSION}

Due to the prevalence of the COVID-19 pandemic, the BAF system has only been tested and driven inside a subdivision, where the speed and vehicular capacity are limited. As a test case scenario, the experiments are done by having a lead and follower vehicles. The lead vehicle varies its speed independently while the trailing vehicle adjusts both its speed and inter-vehicle distance according to the difference between the values shown on the HUD and the actual inputs of the driver. the dashboard camera of the following vehicle is used to record the footage.

The subdivision route highlighted in red in Figure 6 shows the path taken for multiple experiments of the system. This loop is chosen because it has the least amount of widely spaced speed bumps. Such condition allows our testings to experiment on the fastest speed our test vehicle can achieve. These streets are also the widest and has the least number of parked cars at the side.

The actual driving readings are shown in Figure 7. Figure 7(a) shows a screenshot taken from a video demonstrating the HUD system plus the G-force meter application measuring braking and acceleration forces. It can be observed that the sensor is able to detect the cyclist approximately 12 meters away. At the given speed of $24 \mathrm{kph}$, the system calculates a suggested $8 \%$ acceleration input (lower left), while the actual input or the indicated throttle position according to the onboard diagnostics (OBD) system is at $17 \%$. However, the $17 \%$ throttle value is the lowest possible value with the engine running and corresponds to the idle case, i.e., throttle pedal is not depressed. This means that at this point in the video, the driver was not stepping on the throttle. On the other hand, logged OBD data show a maximum throttle position of $81 \%$. This is already the farthest the throttle pedal can move before touching the floor.

Figure 7(b) shows the absence of any significant obstacle directly in the vehicle's path. It can be observed that the system registers 0.01 meters or 1 centimeter, instead of the maximum advertised value of 40 meters. Throughout the entire testing session, the sensor would only be able to consistently measure distances less than 15 meters despite the advertised 40 meter range. When a vehicle goes full braking, the 
vehicle consistently experiences an average force of $1.3 \mathrm{G}$. Occasionally, the experienced G-force is $1.7 \mathrm{G}$, and we have observed that this may be due to the road's unevenness.

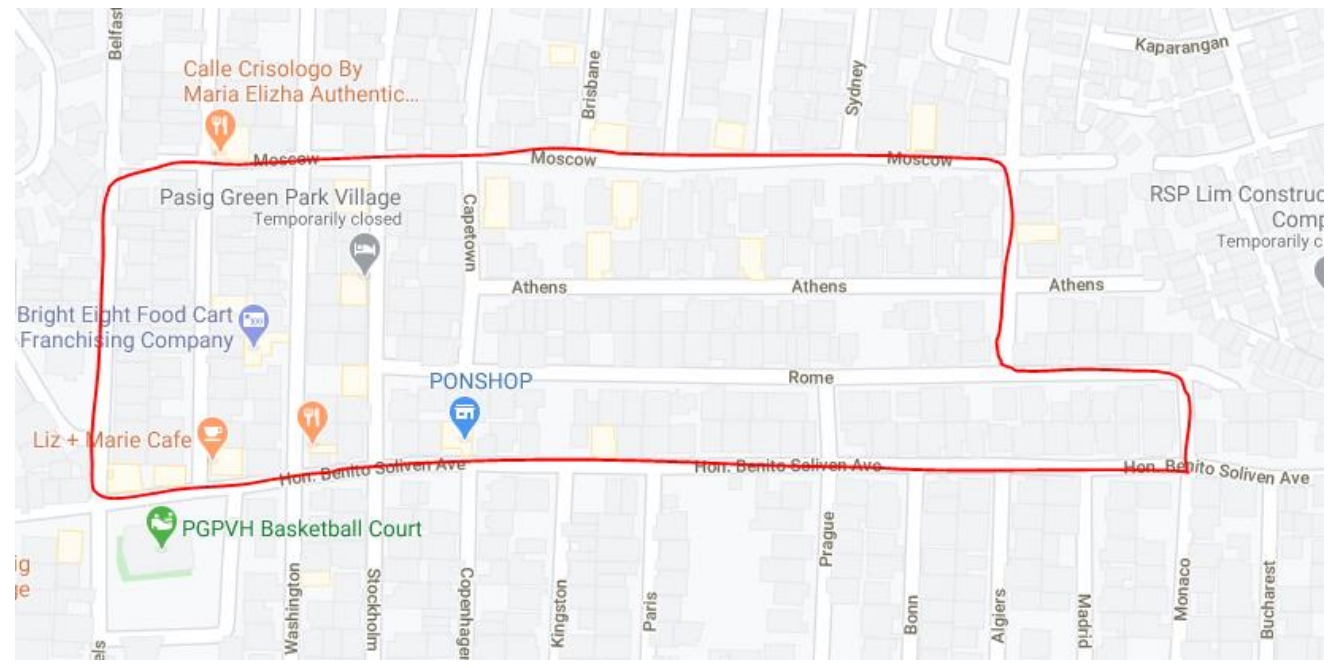

Figure 6. Test route in a subdivision.

Figure 8 shows the gravitational forces in any direction acting on the vehicle when it is in motion. The $\mathrm{x}$-axis (red graph) represents the lateral G-forces and are seen to be minimal since the vehicle is traveling along a straight line at this point in time. The value would increase or decrease when it makes a turn. The $\mathrm{y}$-axis (green graph) represents the vertical G-forces. It is almost constantly reading at $1 \mathrm{G}$, i.e., equal to the force of gravity. This value would change when going up or down slopes. Lastly, the z-axis (blue graph) represents the forward and backward forces. This value constantly changes since the vehicle is always either accelerating or decelerating.

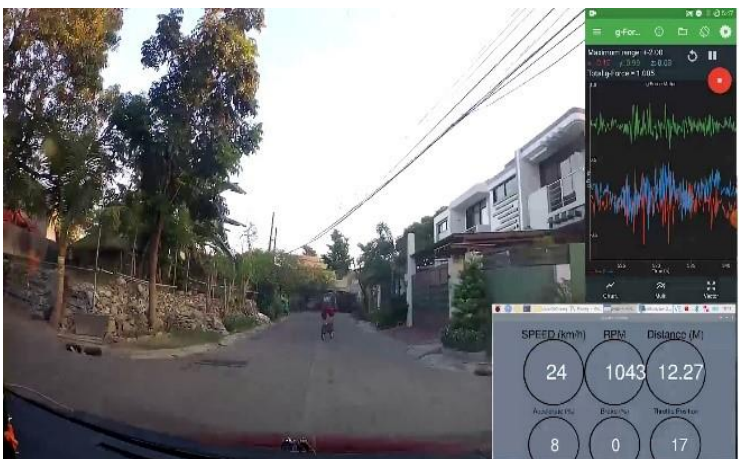

(a)

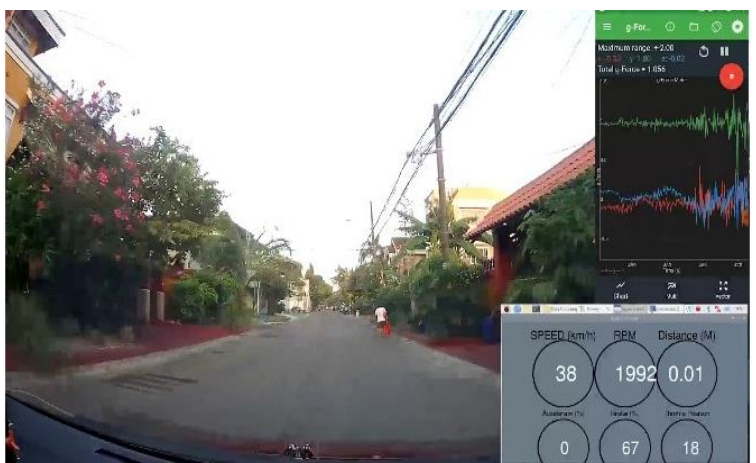

(b)

Figure 7. Actual driving readings (a) actual HUD and G-force meter readings, and (b) No direct obstacles detected in one of the conducted experiments

In Figure 9(a), it must be noted that the $17 \%$ throttle position is the minimum value in which there is no input on the throttle pedal. It can be observed that while there are frequent changes in the level of input, these changes are not abrupt or sudden in increase with respect to the previous value that would warrant abrupt acceleration that would be considered uncomfortable to passengers. In comparison to the calculated throttle input from the BAF, it can be observed that there are some abrupt increases in values in the first half of the graph, and the values appearing to reach a steady state in the second half. However, these abrupt changes may be attributed to the frequent changes in both inter-vehicle distance and vehicle speed as seen in Figure 9(b). 
The vehicle has an initial speed of $25 \mathrm{kph}$ and an initial inter-vehicle distance of 15 meters. It slows down to $5 \mathrm{kph}$ with the distance decreasing to 5 meters because the vehicle in front is slowing down due to a speed bump. The speed can then be seen increasing to safe values seen within a subdivision. It can also be observed that the change in inter-vehicle distance corresponds with the change in speed. This may be due to attempting to maintain a constant inter-vehicle distance but difficult to achieve due to the vehicle in front constantly accelerating. This also appears to reflect the occurrence of phantom traffic jams as both inter-vehicle distance and speed constantly change instead of reaching a steady-state value.

From the observations and gathered data, the performance of the proposed BAF system achieved at most $10 \%$ difference between the values shown in our HUD and the vehicle's speedometer and tachometer. The desired inter-vehicle distance has also been achieved. The BAF system has also aided drivers to observe their driving behaviors including their acceleration and braking patterns.

Figure 10 shows the actual driving derived from one of the experiments (viewed left to right, top to bottom) where the proposed BAF hardware and monitoring system are installed. Incoming vehicles are not detected but only the vehicle in front. Future improvements will include the calibration of the LIDAR sensor and detection of pedestrians walking on the side of the road.

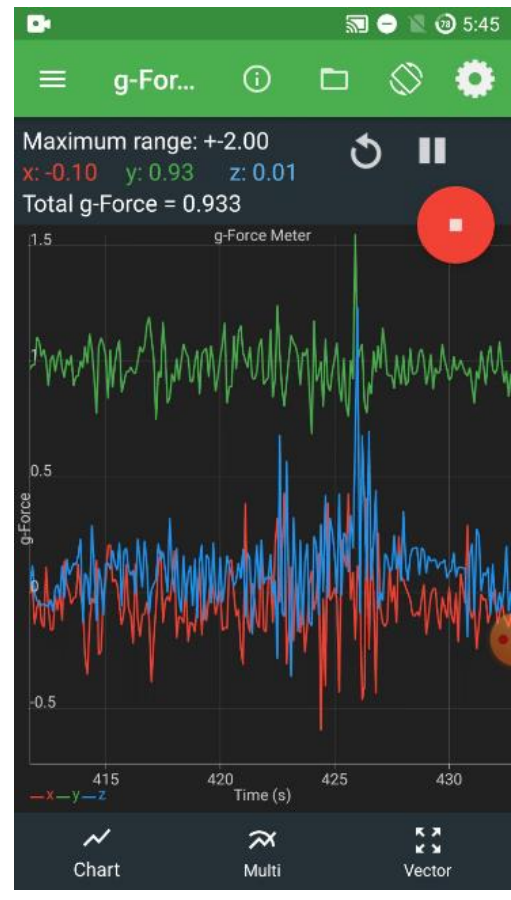

Figure 8. A spike in the G-force (blue) due to hard braking

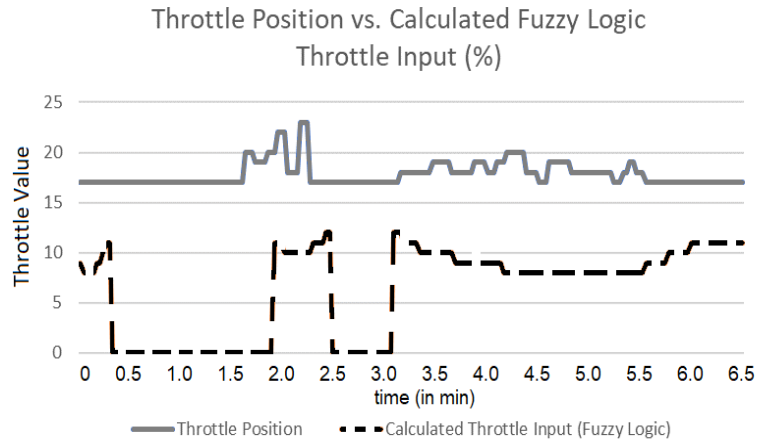

(a)

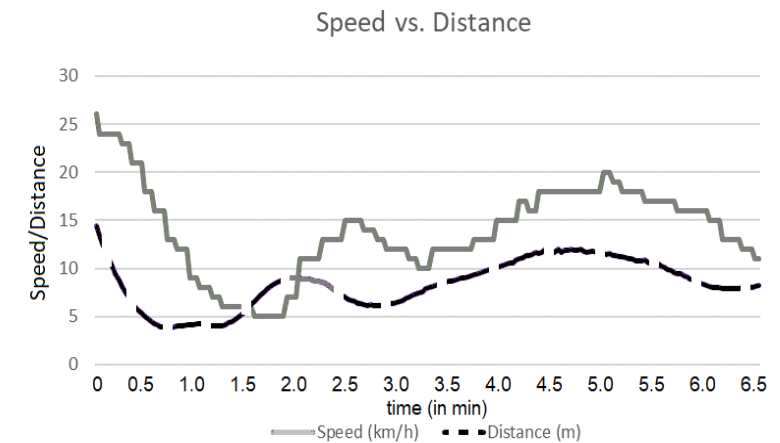

(b)

Figure 9. Experimental driving results (a) throttle position vs. BAF calculated acceleration, and (b) speed $(\mathrm{kph})$ and distance $(\mathrm{m})$ vs sampling instance 

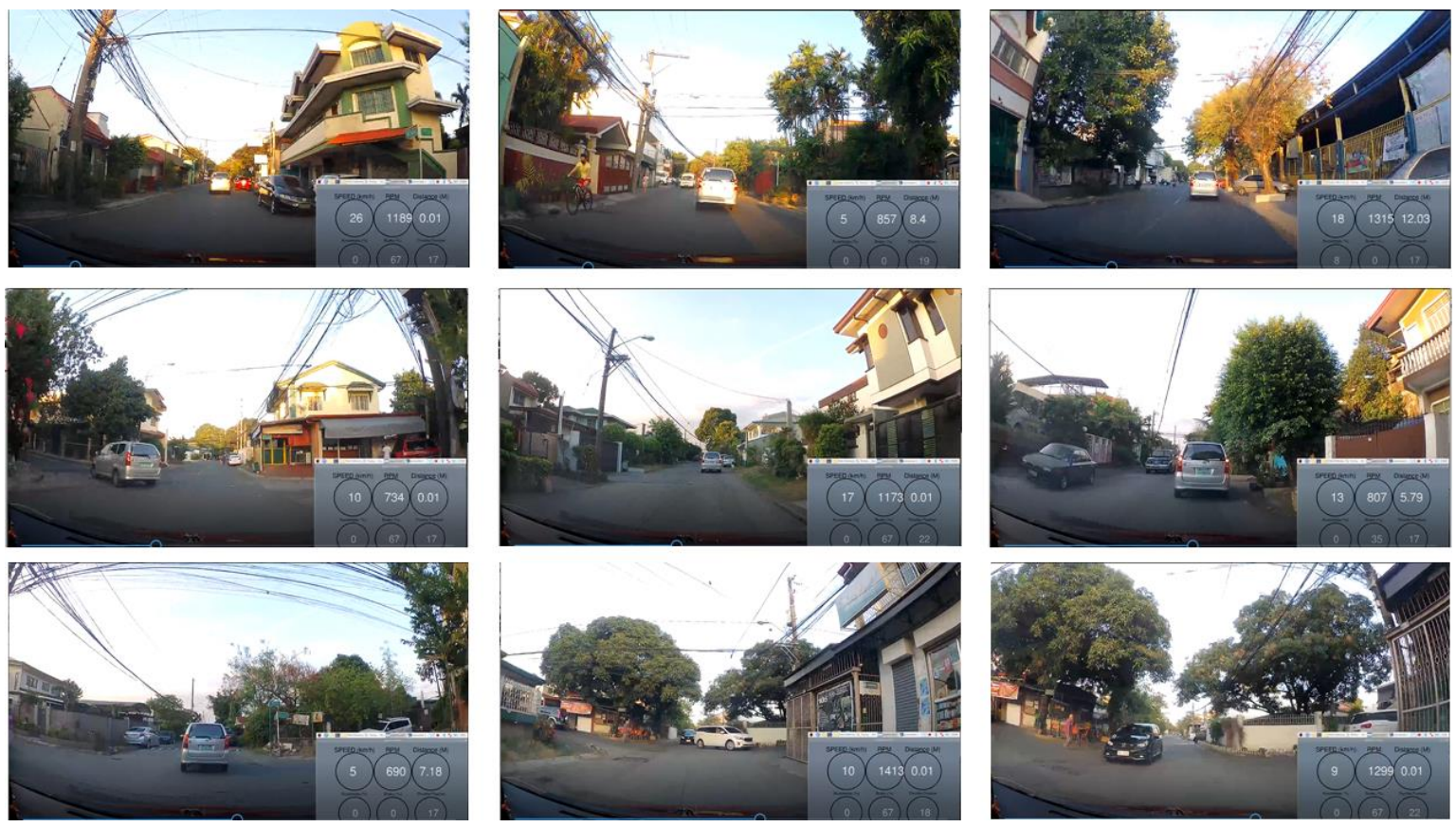

Figure 10. Snapshots taken every 15 seconds when driving the 2008 Toyota Vios $3 \mathrm{~J}$ along the test route shown in Figure 6

\section{CONCLUSION}

In this work, a brake and acceleration feature (BAF) for advanced driver assistance system has been proposed, empirically tested, and verified and can reach an accuracy of $90 \%$ during our test runs in a controlled environment. The BAF hardware is easily integrated and installed in a test vehicle without any major change in the vehicle. This type of installation allows the user to integrate the system in any type of vehicles, as long as the ECU is accessible. With off-the-shelf components, BAF tries to maintain an acceptable distance and speed between leading and trailing vehicles.

In future research directives, the BAF hardware is intended to be placed in a chassis that can withstand sun and rain environment conditions. We also intend to perform continuous and extensive empirical experiments to fully provide a method on how to model a driver's driving patterns and behaviors. Also, 3D environment monitoring will be sensed to further use the capability of the LIDAR. 3D data will allow the vehicle to have a better understanding of its surroundings.

\section{REFERENCES}

[1] L. C. Davis, "Mitigation of congestion at a traffic bottleneck with diversion and lane restrictions," Physica A: Statistical Mechanics and its Applications, vol. 391, no. 4, pp. 1679-1691, Feb. 2012, doi: 10.1016/j.physa.2011.10.036.

[2] F. E. Sancar, B. Fidan, J. P. Huissoon, and S. L. Waslander, "MPC based collaborative adaptive cruise control with rear end collision avoidance," in 2014 IEEE Intelligent Vehicles Symposium Proceedings, Jun. 2014, pp. 516-521, doi: 10.1109/IVS.2014.6856559.

[3] P. Jiang, Z. Dong, Y. Wang, Y. Su, and Z. Li, "Empirical study of phantom traffic jams on urban expressway," in 2018 Ninth International Conference on Intelligent Control and Information Processing (ICICIP), Nov. 2018, pp. 99-102, doi: 10.1109/ICICIP.2018.8606713.

[4] A. Vahidi and A. Eskandarian, "Research advances in intelligent collision avoidance and adaptive cruise control," IEEE Transactions on Intelligent Transportation Systems, vol. 4, no. 3, pp. 143-153, Sep. 2003, doi: 10.1109/TITS.2003.821292.

[5] H. Beglerovic, T. Schloemicher, S. Metzner, and M. Horn, "Deep learning applied to scenario classification for lane-keep-assist systems," Applied Sciences, vol. 8, no. 12, Dec. 2018, Art. no. 2590, doi: 10.3390/app8122590.

[6] H. Iizuka and K. Kurami, Automatic braking system with proximity detection to a preceding vehicle. uS Patent 5,278,764, 1994.

[7] R. A. Daziano, M. Sarrias, and B. Leard, "Are consumers willing to pay to let cars drive for them? Analyzing response to autonomous vehicles," Transportation Research Part C: Emerging Technologies, vol. 78, pp. 150-164, May 2017, doi: 10.1016/j.trc.2017.03.003.

[8] S. Park, H. Rakha, K. Ahn, and K. Moran, "Fuel economy impacts of manual, conventional cruise control, and predictive ecocruise control driving," International Journal of Transportation Science and Technology, vol. 2, no. 3, pp. 227-242, Sep. 2013, doi: 10.1260/2046-0430.2.3.227.

[9] A. H. Eichelberger and A. T. McCartt, "Toyota drivers' experiences with dynamic radar cruise control, pre-collision system, and lane-keeping assist," Journal of Safety Research, vol. 56, pp. 67-73, Feb. 2016, doi: 10.1016/j.jsr.2015.12.002.

[10] J. B. Cicchino and D. S. Zuby, "Prevalence of driver physical factors leading to unintentional lane departure crashes," Traffic Injury Prevention, vol. 18, no. 5, pp. 481-487, Jul. 2017, doi: 10.1080/15389588.2016.1247446. 
[11] K. Shimazaki, T. Ito, A. Fujii, and T. Ishida, "The public's understanding of the functionality and limitations of automatic braking in Japan," IATSS Research, vol. 42, no. 4, pp. 221-229, Dec. 2018, doi: 10.1016/j.iatssr.2017.11.002.

[12] N. C. Basjaruddin, K. Kuspriyanto, S. Suhendar, D. Saefudin, and V. A. Azis, "Hardware simulation of automatic braking system based on fuzzy logic control," Journal of Mechatronics, Electrical Power, and Vehicular Technology, vol. 7, no. 1, pp. 1-6, Jul. 2016, doi: 10.14203/j.mev.2016.v7.1-6.

[13] I. Solís-Marcos, C. Ahlström, and K. Kircher, "Performance of an additional task during level 2 automated driving: An on-road study comparing drivers with and without experience with partial automation," Human Factors: The Journal of the Human Factors and Ergonomics Society, vol. 60, no. 6, pp. 778-792, Sep. 2018, doi: 10.1177/0018720818773636.

[14] C. Gold, F. Naujoks, J. Radlmayr, H. Bellem, and O. Jarosch, "Testing scenarios for human factors research in level 3 automated vehicles," in International Conference on Applied Human Factors and Ergonomics, Jun. 2018, pp. 551-559, doi: 10.1007/978-3319-60441-1_54.

[15] B. Markwalter, "The path to driverless cars (CTA insights)," IEEE Consumer Electronics Magazine, vol. 6, no. 2, pp. 125-126, Apr. 2017, doi: 10.1109/MCE.2016.2640625.

[16] C. Pakusch, G. Stevens, A. Boden, and P. Bossauer, "Unintended effects of autonomous driving: A study on mobility preferences in the future," Sustainability, vol. 10, no. 7, Jul. 2018, Art. no. 2404, doi: 10.3390/su10072404.

[17] A. Herrmann, W. Brenner, and R. Stadler, Autonomous driving. Emerald Publishing Limited, 2018.

[18] Y. Wiseman, "Autonomous vehicles," Encyclopedia of Organizational Knowledge, Administration, and Technologies, 2019.

[19] J. Vergeest and B. van Arem, "The effect of vehicle acceleration near traffic congestion fronts," in 2012 IEEE Intelligent Vehicles Symposium, Jun. 2012, pp. 45-50, doi: 10.1109/IVS.2012.6232152.

[20] K. Osman, M. F. Rahmat, and M. A. Ahmad, "Modelling and controller design for a cruise control system," in 2009 5th International Colloquium on Signal Processing \& Its Applications, Mar. 2009, pp. 254-258, doi: 10.1109/CSPA.2009.5069228.

[21] R. Rizvi, S. Kalra, C. Gosalia, and S. Rahnamayan, "Fuzzy adaptive cruise control system with speed sign detection capability," in 2014 IEEE International Conference on Fuzzy Systems (FUZZ-IEEE), Jul. 2014, pp. 968-976, doi: 10.1109/FUZZIEEE.2014.6891748.

[22] T. Someswari, A. K. Tiwari, and N. R, "A dynamic cruise control system (DCCS) for effective navigation system," International Journal of Electrical and Computer Engineering (IJECE), vol. 10, no. 5, pp. 4645-4654, Oct. 2020, doi: 10.11591/ijece.v10i5.pp4645-4654.

[23] W. Hong-bo, S. Xiao-wen, and Y. Jiang, "Research on hierarchical control of automobile adaptive cruise system based on the mode switching," in 2016 Chinese Control and Decision Conference (CCDC), May 2016, pp. 2733-2738, doi: 10.1109/CCDC.2016.7531446.

[24] G. P. T, “Adaptive cruise control employing taillight tracking for a platoon of autonomous vehicles," International Journal of Advanced Trends in Computer Science and Engineering, vol. 8, no. 3, pp. 640-645, Jun. 2019, doi: 10.30534/ijatcse/2019/48832019.

[25] B. Rao. Yenugula and M. Zia-ur-Rahman, "Stability control structure of hovercraft prototype utilising PID controller," Bulletin of Electrical Engineering and Informatics, vol. 6, no. 4, pp. 348-350, Dec. 2017, doi: 10.11591/eei.v6i4.864.

[26] N. C. Basjaruddin, K. Kuspriyanto, D. Saefudin, and A. Rachman, "Hardware simulation of active lane keeping assist based on fuzzy logic," Indonesian Journal of Electrical Engineering and Computer Science, vol. 5, no. 2, Feb. 2017, Art. no. 321, doi: 10.11591/ijeecs.v5.i2.pp321-326.

[27] E. R. Magsino, M. F. Say, and J. A. Tan, “Achieving complete UAV delivery in the presence of motor failures," in 2020 IEEE 10th Symposium on Computer Applications \& Industrial Electronics (ISCAIE), Apr. 2020, pp. 1-5, doi: 10.1109/ISCAIE47305.2020.9108809.

[28] M. Z. Ismail, M. H. N. Talib, Z. Ibrahim, J. Mat Lazi, and Z. Rasin, "Experimental simplified rule of self tuning fuzzy logicmodel reference adaptive speed controller for induction motor drive," Indonesian Journal of Electrical Engineering and Computer Science (IJEECS), vol. 20, no. 3, pp. 1653-1664, Dec. 2020, doi: 10.11591/ijeecs.v20.i3.pp1653-1664.

[29] W. Pananurak, S. Thanok, and M. Parnichkun, "Adaptive cruise control for an intelligent vehicle," in 2008 IEEE International Conference on Robotics and Biomimetics, Feb. 2009, pp. 1794-1799, doi: 10.1109/ROBIO.2009.4913274.

[30] G. Gunter et al., "Are commercially implemented adaptive cruise control systems string stable?," IEEE Transactions on Intelligent Transportation Systems, vol. 22, no. 11, pp. 6992-7003, Nov. 2021, doi: 10.1109/TITS.2020.3000682.

[31] S. P. Deligianni, M. Quddus, A. Morris, A. Anvuur, and S. Reed, “Analyzing and modeling drivers' deceleration behavior from normal driving," Transportation Research Record: Journal of the Transportation Research Board, vol. 2663, no. 1, pp. 134-141, Jan. 2017, doi: 10.3141/2663-17.

[32] E. R. Magsino and I. W. H. Ho, "An intelligent highway tollgate queue selector for improving server utilization and vehicle waiting time," in 2016 IEEE Region 10 Symposium (TENSYMP), May 2016, pp. 271-276, doi: 10.1109/TENCONSpring.2016.7519417.

\section{BIOGRAPHIES OF AUTHORS}

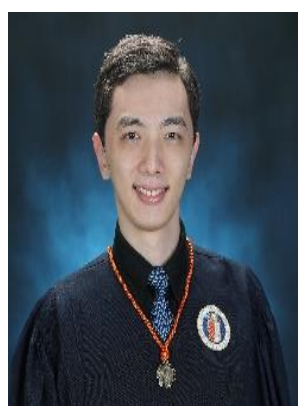

Johann Carlo Marasigan (D) SC SC received his B.S. degree in Electronics Engineering in the Electronics, Computer and Communications Engineering (ECCE) department of the Ateneo de Manila University in Quezon City, Philippines. His research interests include intelligent computing, autonomous vehicles, and renewable energy and embedded systems. His interests mostly relate to computer systems and hardware, specifically microprocessors and other related components. He is currently reviewing in preparation for the upcoming licensure examination of electronics engineers. He can be contacted at email: johann.marasigan86@ gmail.com. 


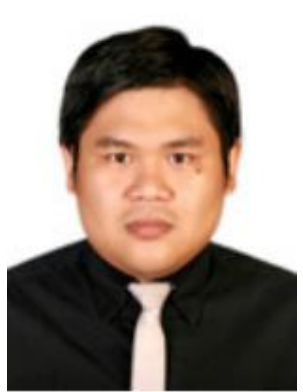

Gian Paolo Mayuga (D) $8 \mathrm{~S}$ SC $\mathrm{P}$ received his B.S. degree in Electronics and Communications engineering and M.S. degree in Electrical Engineering from University of the Philippines Diliman in Quezon City, Philippines. He received his Ph.D. degree in Information Science from Nara Institute of Science and Technology in Nara, Japan in 2016. He has published his research works in various international conferences and journals, such as IEEE and IEICE. Dr. Mayuga is currently an assistant professor at the Electronics, Computer, and Communications Engineering (ECCE) department of Ateneo de Manila University in Quezon City, Philippines. His current research interests include design for reliability and intelligent computing. He is a member of the Institute of Electronics Engineers of the Philippines (IECEP) and IEEE. He can be contacted at email: gmayuga@ateneo.edu.

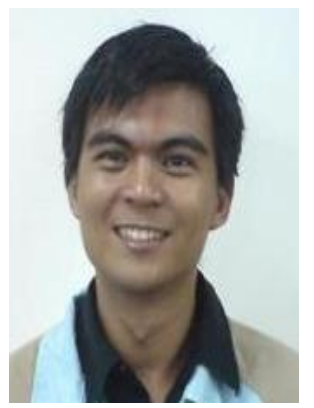

Elmer Magsino (iD) SI SC P received the B.Sc. degree in Electronics and Communications Engineering and M.Sc. degree in Electrical Engineering from The University of the PhilippinesDiliman in 2002 and 2006, respectively. He completed his Ph.D. in Electronic and Information Engineering at The Hong Kong Polytechnic University, Kowloon, Hong Kong in 2021 focusing on data dissemination in vehicular networks. Since 2012, he has published his research studies in various local and international conferences and journals, e.g., IEEE, ACM, IAENG, and IOP. Along with his student, they were awarded as the Best Paper in the 2012 ICCSCE conference. He has also served as reviewers and committee members in various journals and international conferences, respectively. Before undertaking the academic career, Dr. Magsino served as a design engineer in a power electronics company. Currently, he is an Assistant Professor with the Department of Electronics and Communications Engineering (ECE), Gokongwei College of Engineering, De La Salle University Manila, Philippines. Dr. Magsino has also served as the Program Coordinator for the Computer Engineering program of the same department from 20132015. He is also a member of the Institute of Electronics Engineers of the Philippines (IECEP), International Association of Engineers (IAENG), Institute of Electrical and Electronics Engineers (IEEE), and National Research Council of the Philippines (NRCP). He can be contacted at email: elmer.magsino@dlsu.edu.ph. 